\title{
Miniature Multi-Element Antenna for Wireless Communications
}

\author{
Luis Jofre, Member, IEEE, Bedri A. Cetiner, Member, IEEE, and Franco De Flaviis
}

\begin{abstract}
We present a novel broad-band miniature antenna and employ it in a multi-element geometry with diversity capabilities for wireless communications. This antenna (diameter $<0.2 \lambda$ and thickness $<0.06 \lambda$ ) consists of two stacked circular patches that create two cylindrical slots resonating at two slightly different frequencies, fed by a strategically positioned coaxial probe. An extensive parametric study and results for a prototype working at $5.2 \mathrm{GHz}$ are presented. A multi-element geometry with two or four of such elements follows. Microelectromechanical system (MEMS)-based switches located within its geometry can not only change the working frequency of the design, but also activate a particular radiation beam depending on their specific location (resonant slot-aperture or feed line). Simulation results of a four-element antenna with dimensions $0.8 \lambda \times 0.8 \lambda \times 0.06 \lambda$ and a frequency band operation from 5 to $6 \mathrm{GHz}$ are presented and compared to an experimental prototype. Circuit and radiation characteristics are discussed in terms of reconfigurability and diversity capabilities.
\end{abstract}

Index Terms-Broad-band communications, diversity methods, mobile antennas.

\section{INTRODUCTION}

D EPLOYMENT of next-generation wireless networks requires systems with broad-band capabilities in high-mobility environments [1]. Applications range from personal communications to home, car, and office networking. High data rates (100 Mb/s and above), high spectral efficiencies, and stronger fading mitigation have to be achieved.

Due to its particular role, the antenna has to simultaneously satisfy stringent requirements concerning geometrical characteristics (small size, light weight, adaptability to actual platform, and nonobstructive to the user), electrical performance (wide bandwidth, radiation properties, high efficiency, reconfigurability, and suitability for diversity) and manufacturing constraints (low cost, reliability, packaging capabilities). Performance must not be degraded by environment (handset, equipment, or human body) and the design must satisfy radiation safety standards.

In existing built-in antenna schemes, much attention has been paid to planar structures such as microstrip, aperture-coupled,

\footnotetext{
Manuscript received June 26, 2001; revised October 7, 2001. This research was supported in part by the Spanish Government Visiting Scholarship Grant, ref. PR2000-0305 0077531229, in part by the Broadcom Company under MICRO Grant 00-28, and in part by DARPA under Grant MDA972-00-1-0002.

L. Jofre is with the Electrical and Computer Engineering Department, University of California at Irvine, Irvine, CA 92697-2625 USA. He is also with the Department of Signal Theory and Communications, Technical University of Catalonia, Barcelona, Spain (e-mail: ljofrero@uci.edu).

B. A. Cetiner and F. De Flaviis are with the Electrical and Computer Engineering Department, University of California at Irvine, Irvine, CA 92697-2625 USA (e-mail: bedri@uci.edu; franco@uci.edu).

Publisher Item Identifier S 0018-926X(02)05442-X
}

and slot antennas. These planar configurations, though lightweight and low cost generally suffer from narrow bandwidth, low radiation efficiency, or excessive directivity, and excessive size for the new wireless personal applications. Additional effort is needed to further reduce actual geometries in order to obtain miniaturized and electromagneticallyrobust designs (according to fundamental size limits). A robust miniature geometry that can be integrated onto a PC-card, directly embedded into a RF circuit, or combined with microelectromechanical systems (MEMS) for reconfigurable antenna applications [2]-[5] is needed. Different solutions [6] for reducing size, increasing bandwidth, or shaping the radiation pattern, include using high dielectric constant material, shorting planes, stacked configurations, or surface etching.

Consequently, our work focuses on developing antenna geometries small enough to be easily accommodated in mobile communication terminals and devices. The broad-band miniature antenna presented here is close in shape to the dime coin and combines several downsizing techniques while exploiting the range of fundamental limits to optimize the size-bandwidth-efficiency compromise. The planar multilayered geometry consists of two stacked, air-filled or dielectrically embedded, circular patches that create two cylindrical slots resonating at two slightly different frequencies, fed by a strategically positioned coaxial probe. Design landmarks as well as impedance and radiation behavior of this novel antenna will be analyzed in Section II. A $C$-band model of the antenna has been designed, fabricated and tested for validation and prototyping purposes and results will be presented in this paper.

A multi-element architecture based on the described radiating element is presented in Section III. The new geometry consists of several such antenna elements, integrated with a set of MEMS-like switches, located on specific points of the antenna geometry: either into the resonant aperture, to change the working frequency, or into the feeding line, to radiate a particular beam. Simulated results for a four-element structure are presented and its circuit and radiation characteristics are discussed. To validate the previous results, measured return loss and mutual coupling for a first prototype of the four qdime are also presented. Finally, the capability of the antenna to create different beams with various radiation patterns is discussed.

\section{StRucture AND CharacterizATION OF THE Single MiniATURE DiME ANTENNA}

We begin with a comparative review of existing sizereduction techniques. We then discuss the design process 
TABLE I

Contrast of Main Planar Antenna Size Reduction TeChNiques

\begin{tabular}{l|c|c|c}
\hline Performances $\Rightarrow$ & $\begin{array}{c}\text { Geometrical: } \\
\text { Size Reduction } \\
\text { with respect to } \\
\text { Resonant Size }\end{array}$ & $\begin{array}{c}\text { Circuital: } \\
\text { Impedance } \\
\text { Bandwidth }\end{array}$ & $\begin{array}{c}\text { Radiation Pattern: } \\
\text { Symmetry vs } \\
\text { Low Cross } \\
\text { Polarization }\end{array}$ \\
\hline $\begin{array}{l}\text { Multilayered: } \\
\text { fold, bend, stack, etc }\end{array}$ & $60 \%$ & $10 \%$ & High \\
\hline $\begin{array}{l}\text { Patch Etching: } \\
\text { slits, slots, notch, etc }\end{array}$ & $50 \%$ & $7 \%$ & Medium \\
\hline $\begin{array}{l}\text { Shorting Planes: } \\
\text { walls, pins, etc }\end{array}$ & $70 \%$ & $5 \%$ & Low \\
\hline $\begin{array}{l}\text { Material: } \\
\text { high dielectric, slow-wave, etc }\end{array}$ & & $2 \%$ & High \\
\hline
\end{tabular}

including concepts for the new structure, the parametric characterization process and the practical aspects of the proposed antenna.

\section{A. Downsizing Design}

During the last few years, several types of planar antennas have been studied and quite a large number of related designs can be found in the literature [6], [7]. Each solution is intended to optimize a certain parameter, such as frequency bandwidth, size, flatness, radiation pattern, polarization, etc. A number of interesting designs exist addressing some of these issues: folded configurations [8]-[11], surface etching techniques [12]-[16], shorting walls or pins [17], [18], or high dielectric constant materials [19], [20]. However, there is difficulty in obtaining good electrical performance (bandwidth, radiation pattern, gain) when reducing size, due to the minimum radiation $\mathrm{Q}$ attainable, associated with their actual field distribution, in accordance with the radiation fundamental limits for small antennas [21], [22].

In order to overcome this size-bandwidth limitation, we examine the basics of electromagnetic foundations for small antenna behavior and how different existing geometries affect it. Table I classify the existing downsizing techniques and summarizes their basic geometrical, circuital and radiation characteristics. From this insight, we derive the following basic design guidelines.

- A multilayered structure to obtain a double resonant behavior.

- Slit etching as a way of increasing the electrical path while maintaining physical size.

- Short-circuiting walls to utilize the half-size reduction factor.

- Dielectric loading, especially in packaged applications, for further size reduction at the cost of lower efficiency.

In our case, a two-layer structure with air as filling material was selected in order to get small size while keeping a good bandwidth and a practical design. Packaged solutions may employ a higher permittivity dielectric [23].

Additionally we have chosen the following.

- A circular geometry to achieve the best volumetric modal distribution in accordance with the minimum $Q$ fundamental limit.
- A slot-radiating aperture to take advantage of the greater bandwidth of slot antennas-compared to the microstrip patch configuration-and its broader radiation pattern.

\section{B. Dime Antenna Geometry}

The proposed antenna geometry and its equivalent circuit diagram are shown in Fig. 1. Its circular shape and size earn it its proposed name dime antenna [24]. It consists of two stacked circular patches, upper and lower, over a ground plane, with a circumference close to $\lambda / 2$. We propose a volumetric geometry different from that of a printed antenna structure. A sectorial slit, with $\theta_{s}$ being the slit angle, is etched into the lower patch to reduce its size and control the impedance matching level. This structure forms two planar radial transmission lines formed by three planes (upper patch, lower patch, and ground plane) as shown in Fig. 1. The top transmission line starts at the coaxial feed point and is limited by the upper-cylindrical slot with length $S_{u}$ and its sectorial wall (or shorting wall) of length $w_{u}$. Likewise for the bottom one, lower-cylindrical slot with length $S_{l}$ and sectorial wall of $w_{l}$. The upper and lower layers are of thickness $h_{u}$ and $h_{l}$, respectively. A coaxial probe, with a characteristic impedance $Z_{0}$, simultaneously feeds the two cylindrical slot antennas through two short transformers made out of the two radial-like transmission lines described above with input impedances $Z_{\mathrm{irl}, u}$ and $Z_{\text {irl }, l}$ [Fig. 1(d)]. The inner conductor of the coaxial feed line is attached to the lower patch passing through a hole on the ground plane to which the outer conductor is connected. The sectorial slit etched into the lower patch forces current to travel from the feed point along the circular slit and down to the ground level through the lower shorting wall. The placement of the coaxial inner conductor $\left(d_{m}, s_{m}\right) s_{m}$ being the distance from the circular patch center and the length of the sectorial slits $\left(\theta_{s}\right)$ are key parameters in achieving good input matching. The lengths $S_{u}$ and $S_{l}$ and the length of the feed point-to-ground path define the resonant frequencies. Detailed discussion on how these parameters affect antenna performance will be given in the next section.

This structure utilizes stacking (two layers), shorting wall (two sectorial walls), and etching (circular slit on the lower patch) techniques to achieve a good size-bandwidth compromise. It radiates as two stacked circular slots, electrically equivalent to a vertical electric dipole, producing an almost 

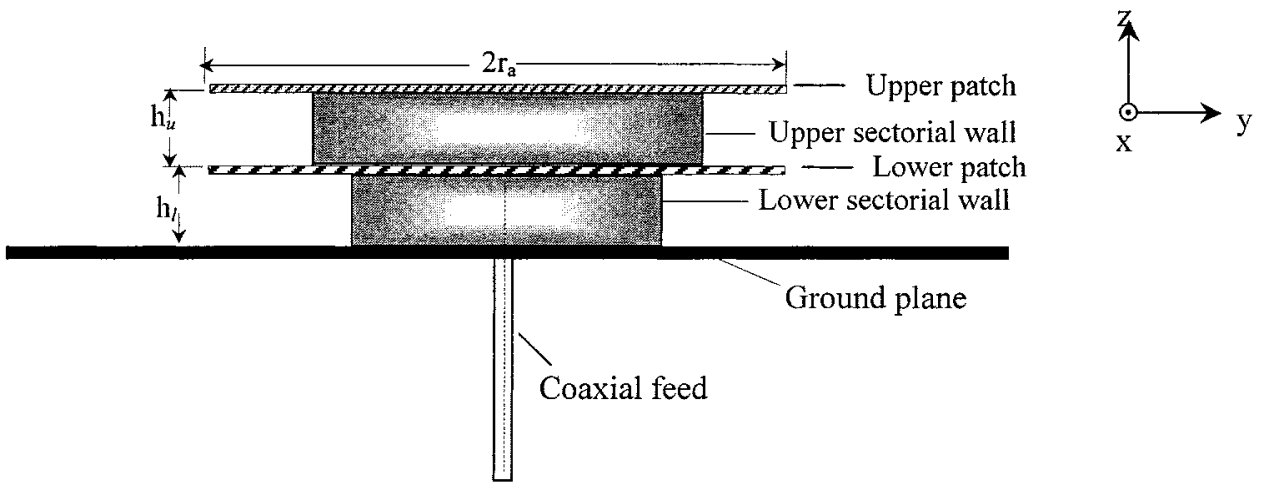

(a)

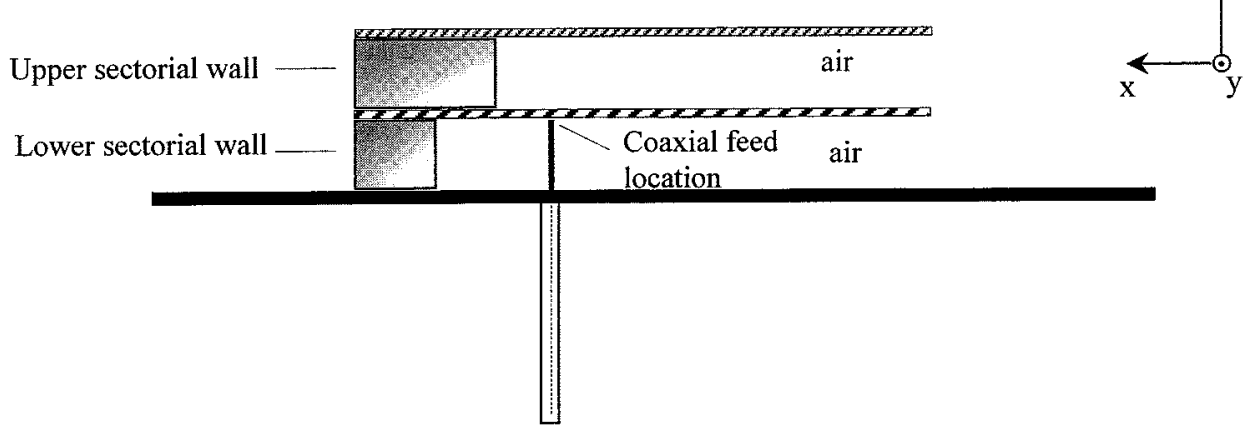

(b)

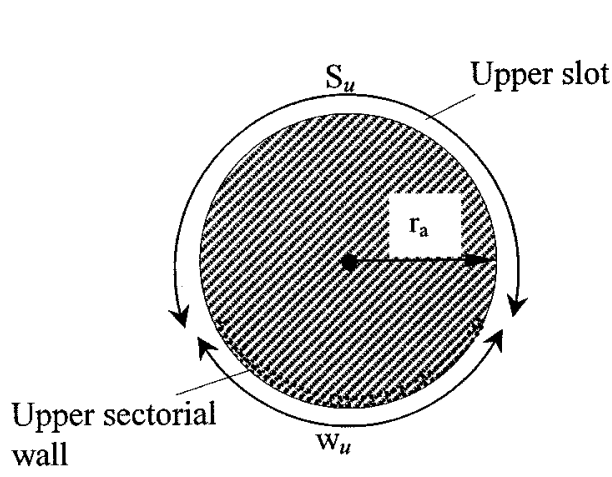

Upper patch

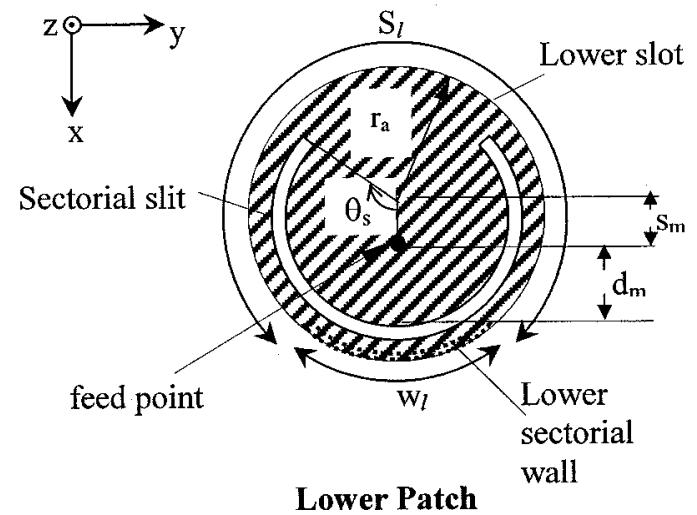

(c)

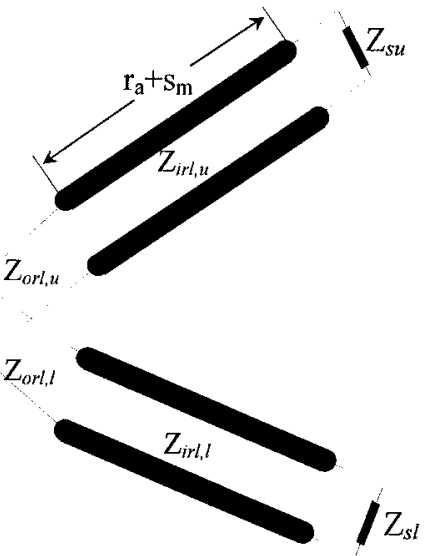

(d)

Fig. 1. Geometry of the proposed dime antenna. (a) Back view. (b) Side views. (c) Top views of the patches. (d) Equivalent circuit diagram. 


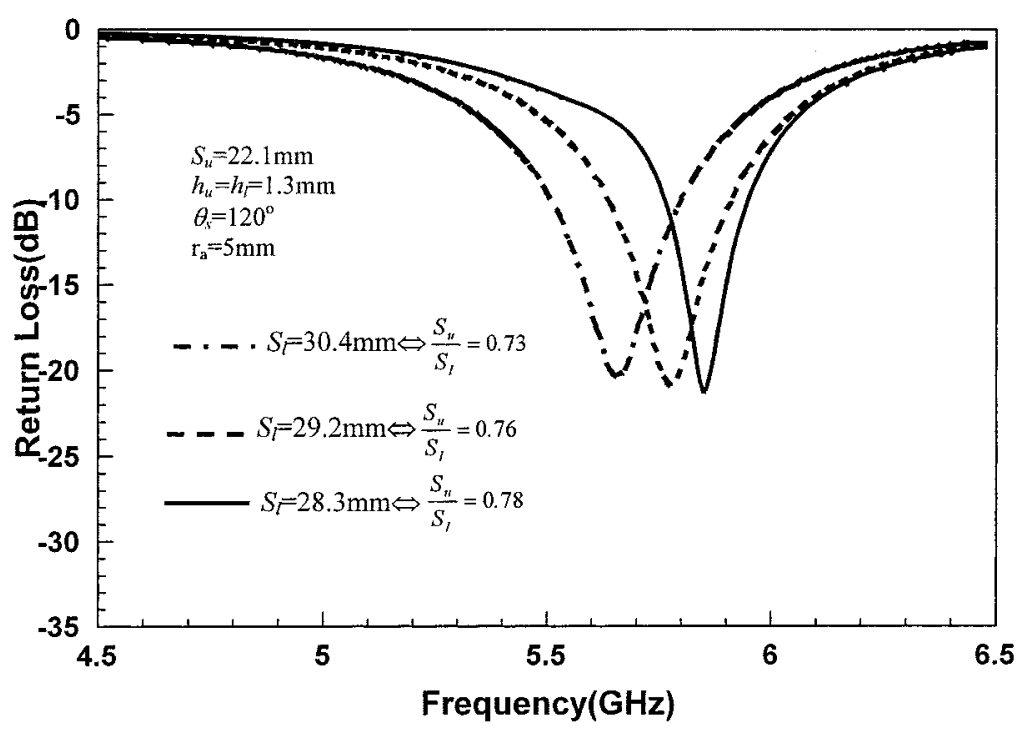

Fig. 2. Return loss of the dime antenna as a function of the lower slot length $S_{l}$.

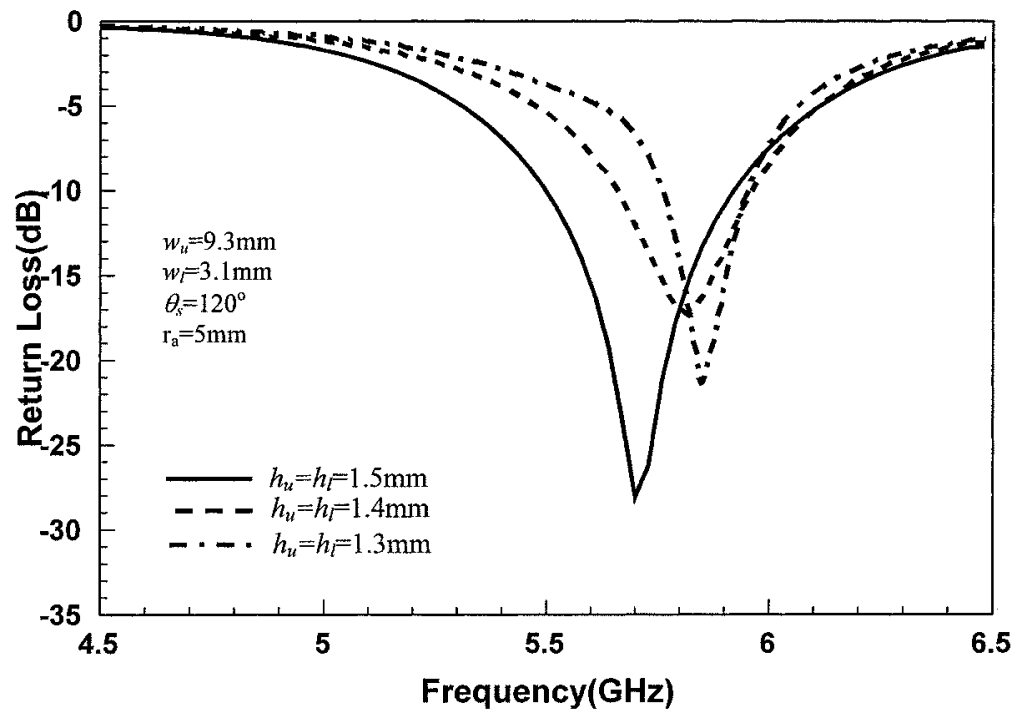

Fig. 3. Return loss of the dime antenna as a function of the layer thickness, $h_{u}=h_{l}$.

omnidirectional pattern that is slightly upper-tilted due to the stacked structure and shorting walls.

\section{Characterization of the Antenna}

Due to its complex geometry, a full-wave technique is required to accurately describe current and field distributions on the antenna. A series of simulations based on the finite-element method (Ansoft HFSS7) have been carried out to investigate how sensitive resonant frequency, bandwidth, and input impedance are to the different physical parameters of the antenna. Figs. 2-4 show how different slot length, layer thickness, and sectorial slit angle affect antenna performance. Fig. 2 shows that resonant frequency can be tuned by changing slot lengths. The ratio of upper to lower slot length can be chosen to achieve either single or dual-band behavior. Fig. 3 shows a $5 \%$ to $10 \%$ increase in bandwidth when the thickness goes up from 1.3 to $1.5 \mathrm{~mm}$. This is in accordance with fundamental radiation laws that predict a wider bandwidth due to a higher consumption of the volume fraction of the sphere enclosing the antenna [25]. Finally, Fig. 4 shows the effect of slit length on the input impedance of the antenna.

To validate the simulation, a test antenna at $5.2 \mathrm{GHz}$ intended for the next-generation Bluetooth frequency band was fabricated and its circuital and radiation parameters were measured (see Fig. 5 for the specific dimensions). The antenna was built by etching a 0.3 -mm-thick copper metal sheet to obtain the three pieces (upper, lower, and ground) of the antenna. These were then soldered together with vertical walls to form the three-dimensional (3-D) miniature structure. Very good agreement between simulated and measured results for the return loss of the antenna are presented in Fig. 5. For a 1:2 VSWR, 12\% bandwidth is achieved and preliminary dual band behavior is observed. In order to characterize antenna coverage, its radiation pattern was numerically simulated and experimentally measured. Fig. 6 shows comparative results for 


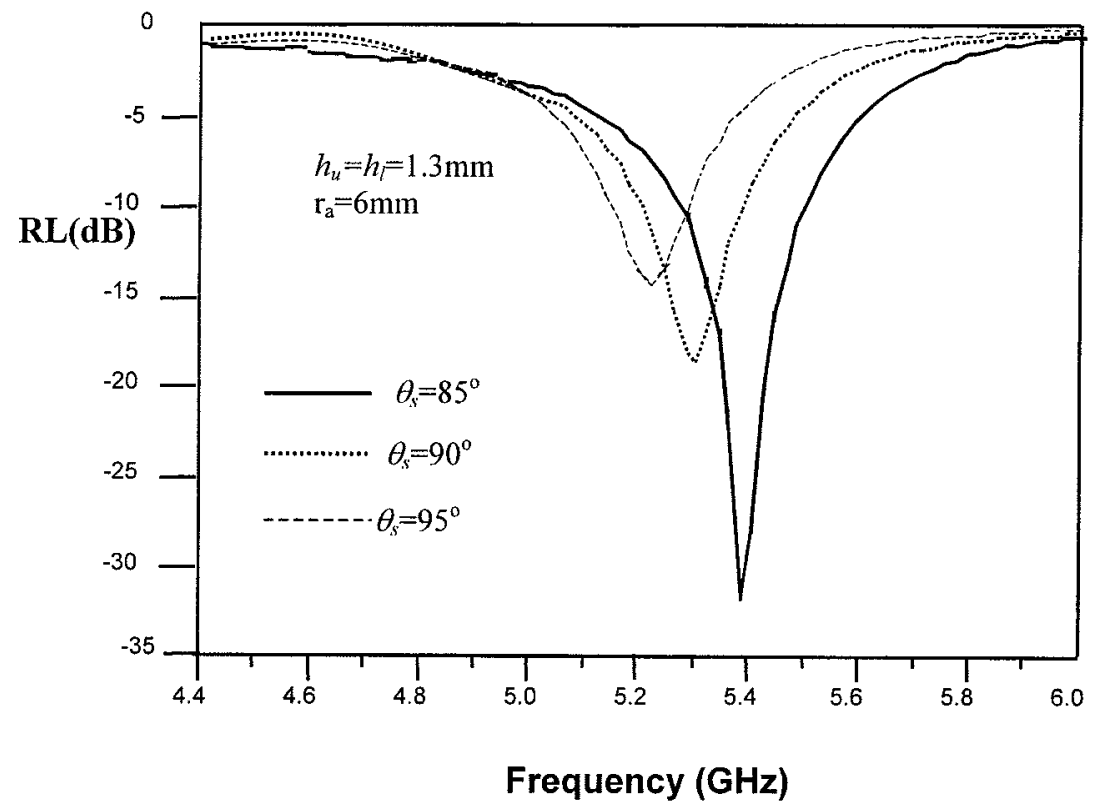

Fig. 4. Return loss of the dime antenna as a function of the sectoral slit angle $\theta_{s}$.

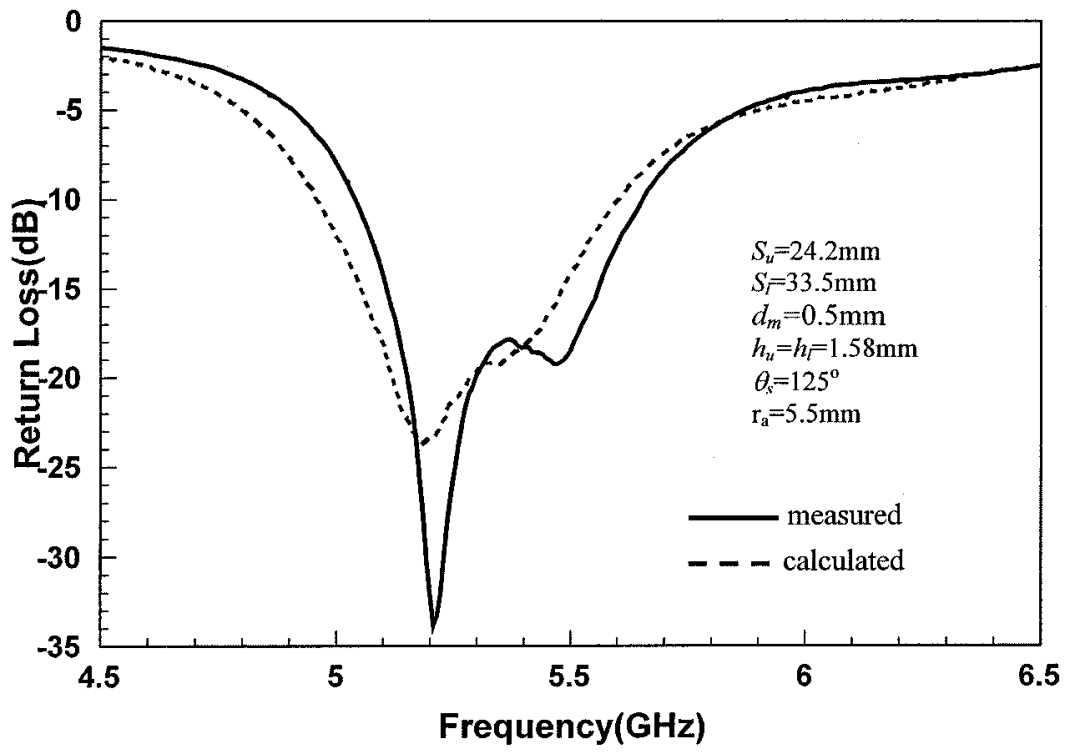

Fig. 5. Comparison of calculated and measured return loss for the fabricated dime antenna.

the E-plane ( $x-z$ plane) and H-plane ( $x-y$ plane) radiation patterns, with very good agreement for both copolar $(\theta)$ and crosspolar $(\phi)$ components. In accordance with its geometry and size, the antenna exhibits a very uniform radiation pattern close to elemental dipole behavior, which makes it suitable for broad coverage applications. Finally, we performed a gain measurement [26] using two different reference antennas, a $\lambda / 2$ dipole and a $\lambda / 4$ monopole, which consistently gave a maximum gain of $2 \mathrm{dBi} \pm 0.2 \mathrm{~dB}$.

Based on the results from the numerical and experimental parametric studies, it is possible to define an antenna-design procedure, consisting of three main steps. In the first step, as seen in Fig. 2, the lower and upper resonant frequencies, $f_{r 1}$ and $f_{r u}$, based on the $\lambda / 2$ resonance of the two vertical slots, fix the main dimensions of the antenna $S_{l}, S_{u}$, and $r_{a}$, according to the following equations:

$$
\begin{aligned}
S_{l} & \cong \frac{c}{2 f_{r 1}} \\
S_{u} & \cong \frac{c}{2 f_{r u}} \\
r_{a} & =\frac{S_{l}+w_{l}}{2 \pi} .
\end{aligned}
$$

Single broad-band or dual-band behavior can be obtained as needed by changing the relative distance between the two resonances.

In the second step, as seen in Fig. 3, the thickness of the two layers fixes the bandwidth of the antenna as follows. 


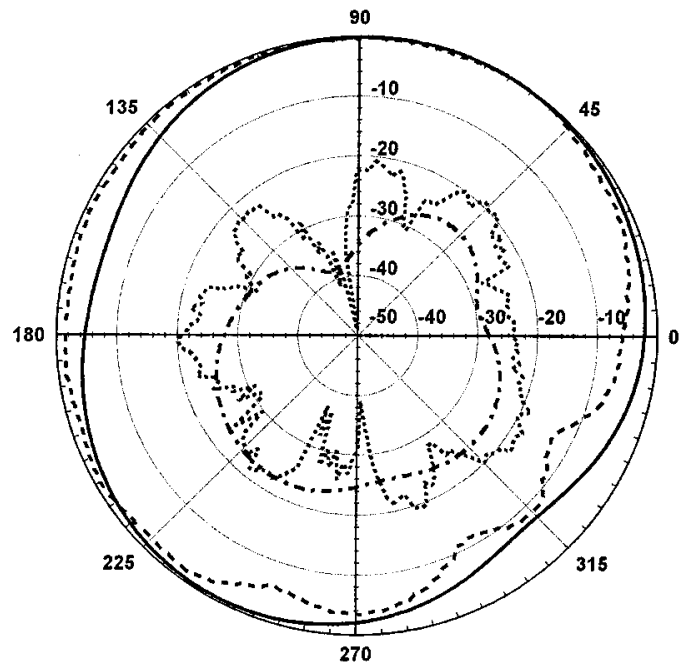

(a)

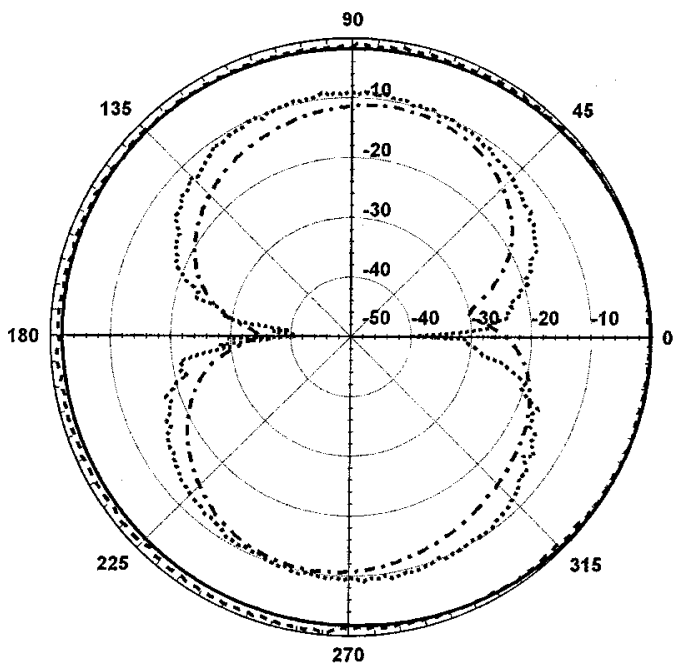

(b)

Co-pol (calculated)

-..... Co-pol (measured)

-....- Cross-pol (calculated)

.................... Cross-pol (measured)

Fig. 6. Comparison of calculated and measured radiation pattern for the fabricated dime antenna. (a) E-plane. (b) H-plane.

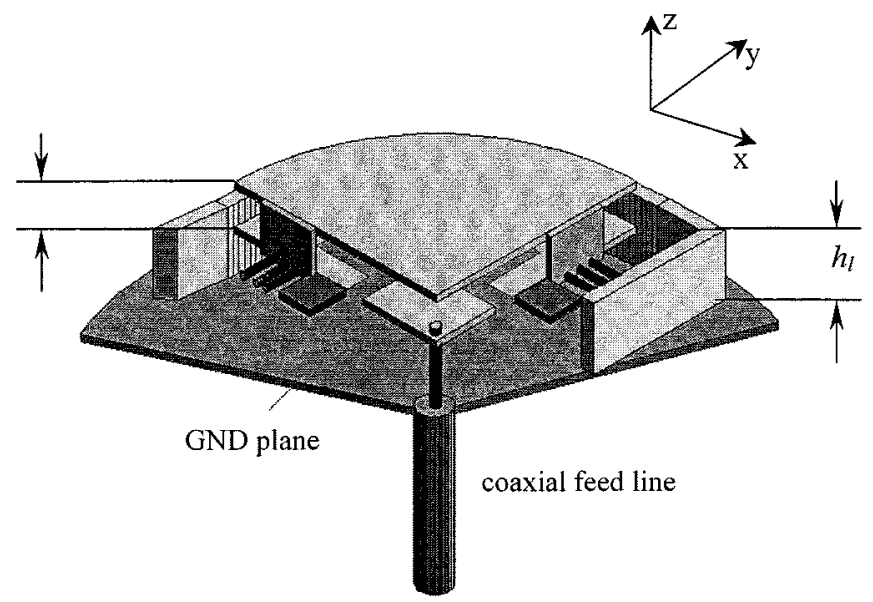

Fig. 7. Architecture of the qdime antenna.

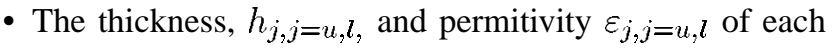
of the two layers fixes the characteristic impedance, $Z_{o r l, j=u, l}$, of the lower and upper radial transmission lines. The purpose of each radial-like transmission line is to match, as a short-line transformer, the higher input impedance of the ending slot antenna $Z_{s j, j=u, l}$ to the input impedance $Z_{i r l, j=u, l}$ of each of the two radial-line parallel layers as seen from the feed point. In order to get an initial guess for $h_{j}$ based on the equivalent circuit shown in Fig. 1(d) and on the characteristic impedance of a radial transmission line [27] conditioned to our geometry with a shape between the rectangular stripline and the radial line, we can formulate the following rough approximate relationship among our different parameters:

$$
h_{j} \cong \frac{\varepsilon_{r, j} \cdot r_{a} \cdot\left(\pi-\theta_{s}\right) \cdot \sqrt{Z_{s j} Z_{i r l, j}}}{240 \pi} .
$$

- The final value for $h_{j}$ is determined by the requirements on frequency bandwidth and radiation efficiency. A fundamental relationship [24] between the diameter $2 a$ of the sphere confining the small antenna, its maximum fractional bandwidth $B W$ and radiating efficiency $\eta$ can be written

$$
B W \cdot \eta \cong(k a)^{3}
$$

where $k$ is the wavenumber. This maximum $B W$ value has to be understood for every individual resonance in the antenna. Therefore, for double resonance geometries, as the dual band dime antenna, this upper limit has to be applied to each individual resonance. When the two resonances are close enough to form a single band, due to the electromagnetic coupling mechanism between the two radiating slots, bandwidths larger than the predicted above for a single resonance can be attained. The radiation efficiency of the 3-D dime antenna will be close to unity since losses are strongly related to the quality factor of the dielectric making up and surrounding the antenna, which in this case is air.

- Based on the two previous steps a good matching-bandwidth compromise for the antenna can be obtained when $h_{j}$ lies in the range $0.15<h_{j} / r_{a}<0.35$. 


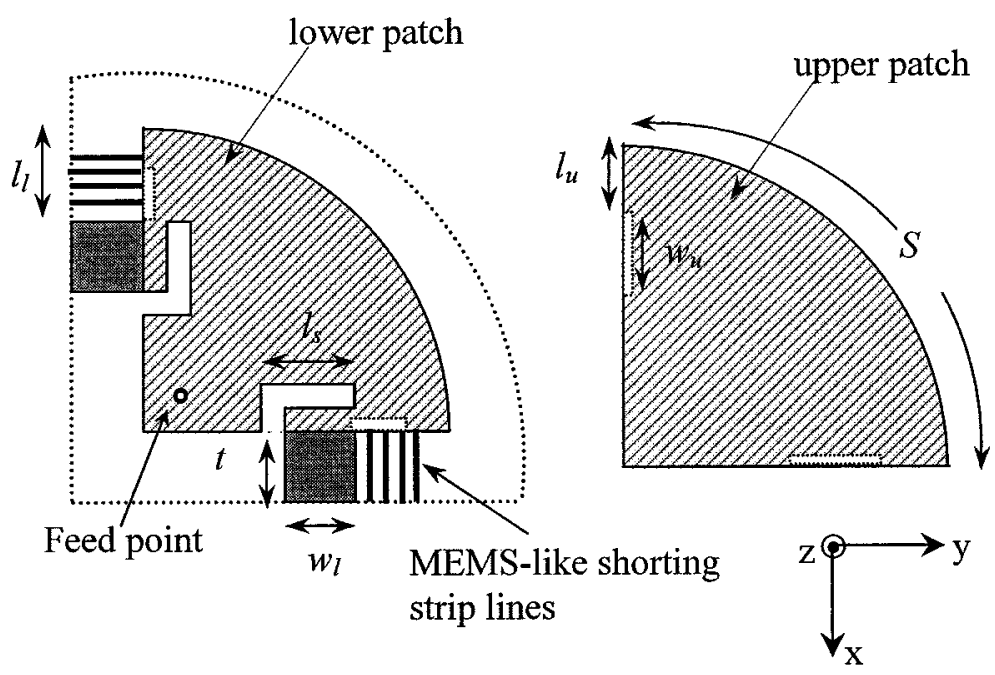

Fig. 8. Top views of the lower and upper patches of the qdime antenna.

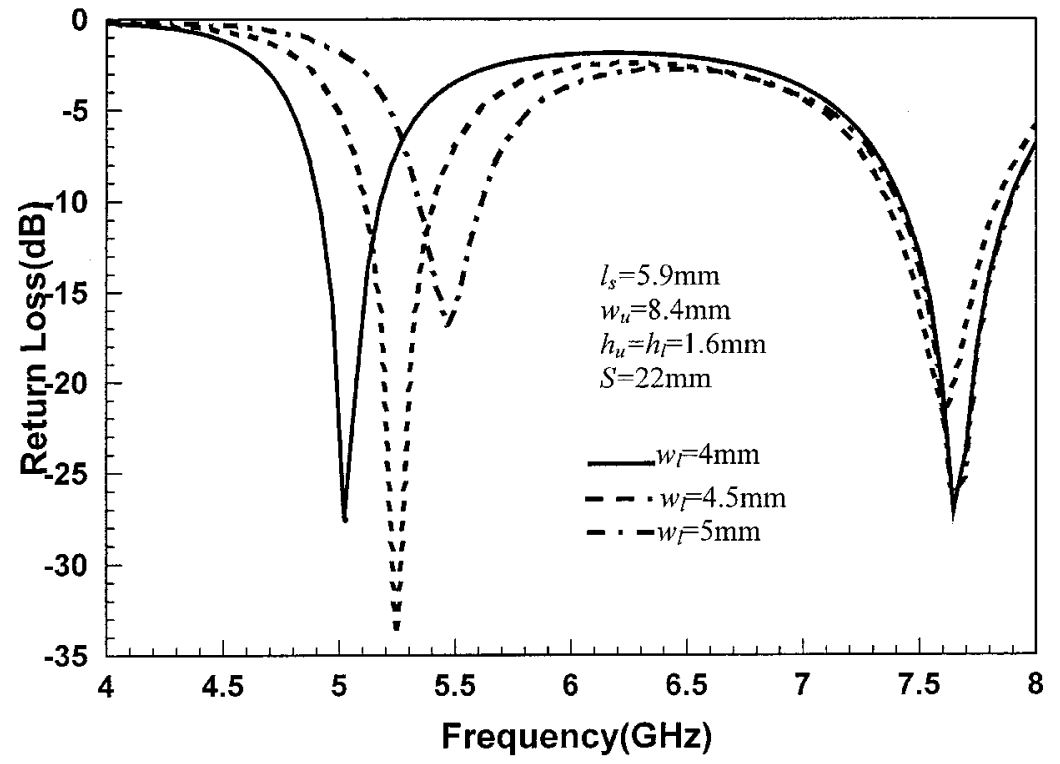

Fig. 9. Return loss of the qdime antenna as a function of $w_{l}$.

In the final step, the dimension of the slit etched in the middle patch, as shown in Fig. 4, fixes the overall impedance matching of the antenna. The angle $\theta_{s}$ is chosen such that the total electrical path between the feed point and the ground plane is $\lambda / 4$, which is approximately

$$
r_{a}\left(1+\theta_{s}\right) \cong \frac{\lambda}{4}
$$

\section{Multi-Element Miniature Antenna}

In this section we discuss the design of a novel multi-element architecture based on the geometry presented previously.

\section{A. The Miniature Single Qdime Antenna}

The geometry of the dime antenna described above is changed slightly to optimize it for a diversity system [28], [29]. This new geometry, called quarter-dime antenna (qdime), is shown in
Fig. 7 and is obtained by square-shaping the back of the antenna to better fit the four-element geometry. Due to the intermediate ground plane connection of the two squared back faces, it also has the advantage of having less mutual coupling and correlation factor when used in a multi-element antenna system capable of providing separate channels for diversity combining.

Like the dime antenna, its structure consists of two planar transmission lines formed by three planes (upper quarter patch, lower quarter patch and ground plane) ending on two radiating slots, as in Fig. 7. In contrast to the dime antenna however, the lower slot is horizontal to accommodate MEMS switches. Two vertical walls with lengths $w_{u}$ and heights $h_{u}$ connect lower and upper patches and determine upper slot length, $S_{u}=S+$ $2 l_{u}$ and upper layer height $h_{u}$ (Figs. 7 and 8). Two horizontal walls with lengths $w_{l}$ and widths $t$ connect the lower patch layer to the ground plane. These walls go through the vertical wall surrounding the lower patch and stretch from ground level to lower patch level with a height of $h_{l}$. This surrounding vertical 


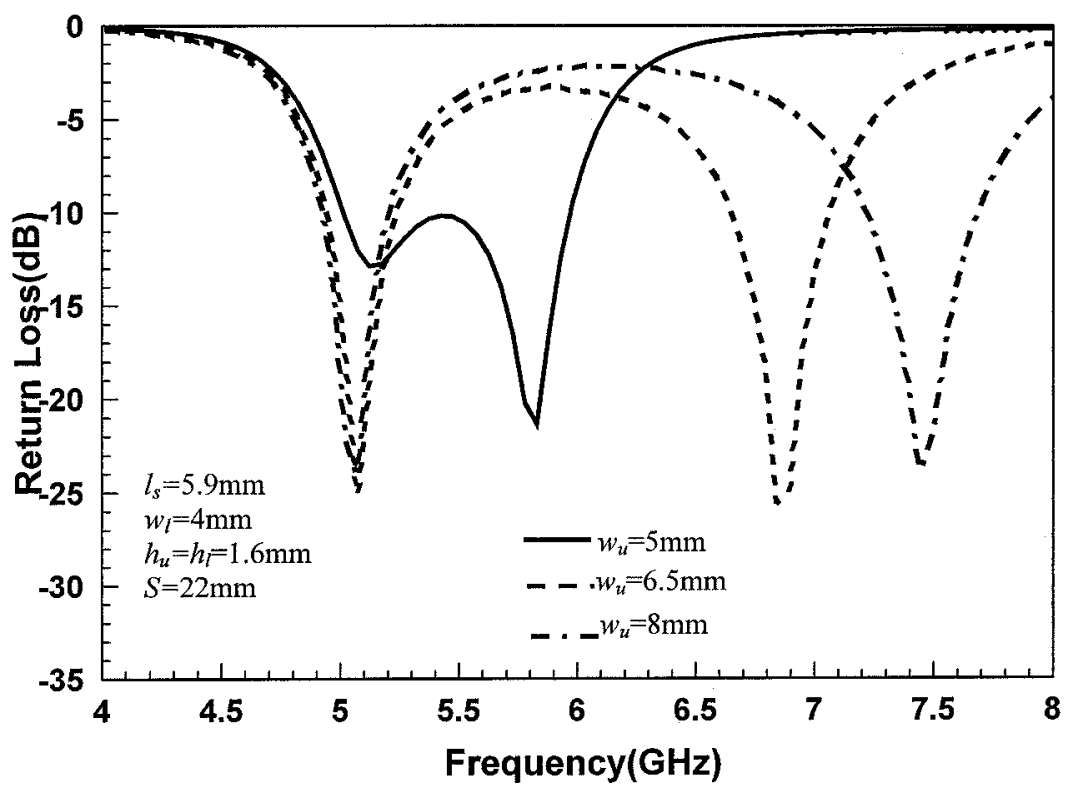

Fig. 10. Return loss of the qdime antenna as a function of $w_{u}$.

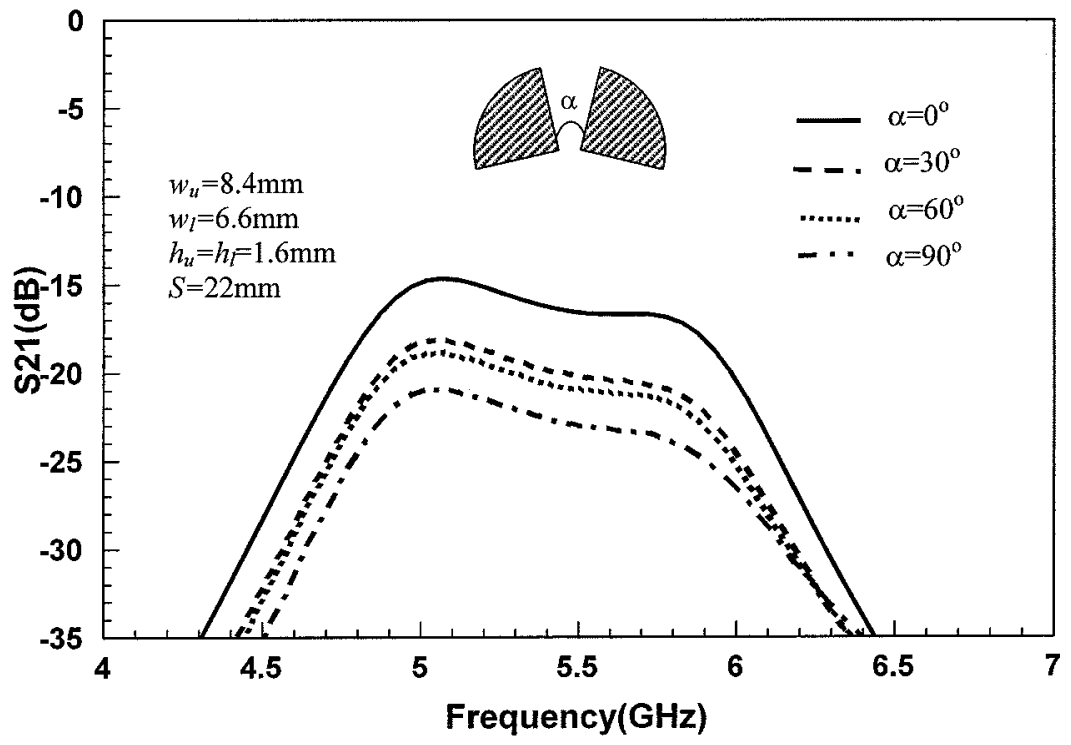

Fig. 11. Comparison of mutual coupling as a function of orientation angle $\alpha$ between two qdime antennas.

wall forms a horizontal lower slot where the horizontal component of the electric field is confined and where the MEMS switches will be located. The length of the horizontal lower slot, $S_{l}=S+2 l_{l}$, is determined by the horizontal wall length $w_{l}$. The slit structure of length $l_{s}$ etched into the lower patch increases the effective electrical length of the antenna while decreasing resonant frequency. This allows miniaturization without sacrificing a good matching performance.

First we investigate the effects of key design parameters such as lower slot length $S u\left(w_{u}\right)$, upper slot length $S_{l}\left(w_{l}\right)$ and slit length $l_{s}$ on antenna performance. Similar to the dime antenna, an appropriate choice of $l_{s}, S_{l}$, and $S_{u}$ effectively tunes the antenna for dual band or single broad-band operation. This tuning behavior can be clearly seen in Fig. 9, where the length of the horizontal walls $w_{l}$ controls the lower resonant frequency. As $w_{l}$ is increased (or lower slot length $S_{l}$ is decreased) the resonant frequency increases as expected, while the higher resonant frequency and its matching level remain unaffected. Similar results are obtained by varying $w_{u}$ while keeping other parameters fixed. Fig. 10 shows that $w_{u}$ controls the higher resonant frequency. We note that when $w_{u}$ is chosen close to $w_{l}$ (or upper slot length $S_{u}$ is close to lower slot length $S_{l}$ ), the antenna has single broad-band behavior. A bandwidth in the order of $20 \%$ is achievable for the frequency band of interest $(5-6 \mathrm{GHz})$.

\section{B. The Four Qdime Reconfigurable Antenna}

As a second step toward multi-element geometry, the interaction between two close identical qdime elements is studied. A numerical analysis has been carried out to study the sensitivity of input impedance and mutual coupling to the relative angular 


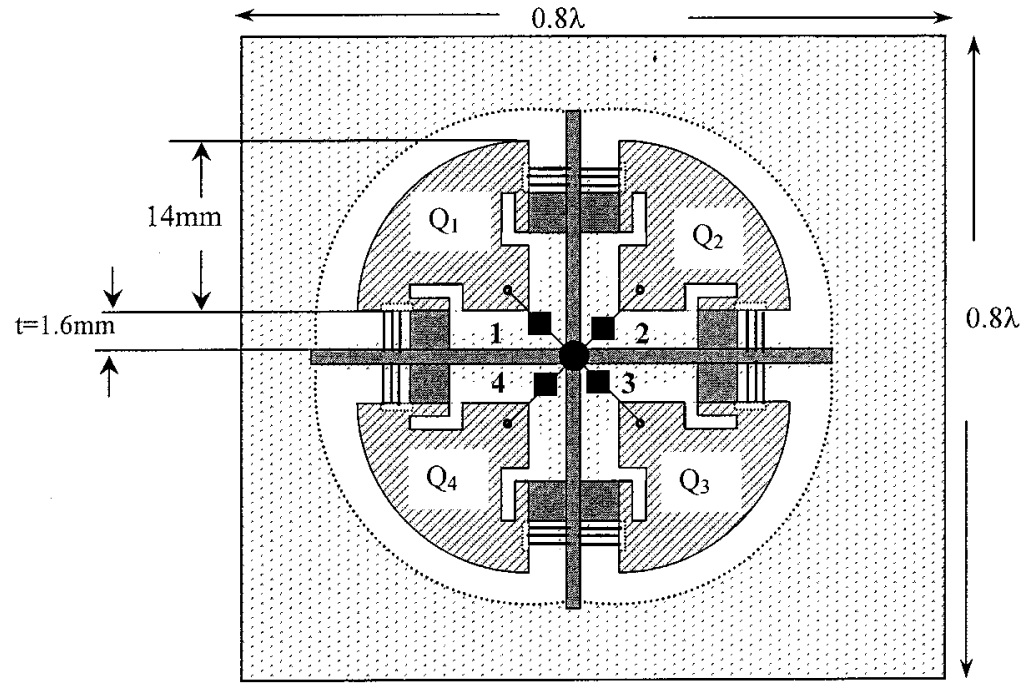

Fig. 12. Top view of the lower-patch plane of the MEMS switch-tunable four-element qdime antenna configuration. $Q_{1}, Q_{2}, Q_{3}, Q_{4}:$ qdime antennas. $1,2,3$, 4: MEMS switches. ( $\left.w_{u}=5 \mathrm{~mm}, w_{l}=4 \mathrm{~mm}, l_{s}=5.9 \mathrm{~mm}, h_{u}=h_{l}=1.6 \mathrm{~mm}, S=22 \mathrm{~mm}\right)$.

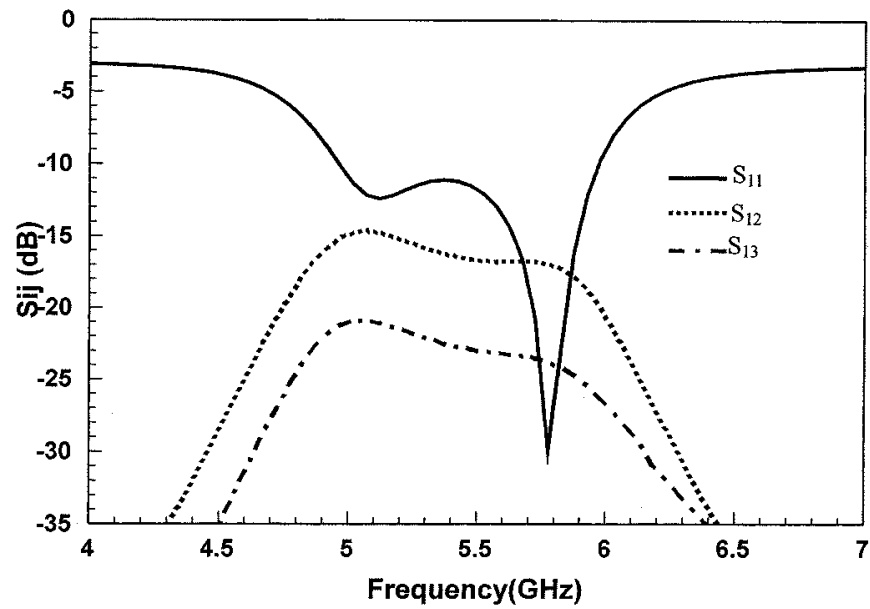

(a)

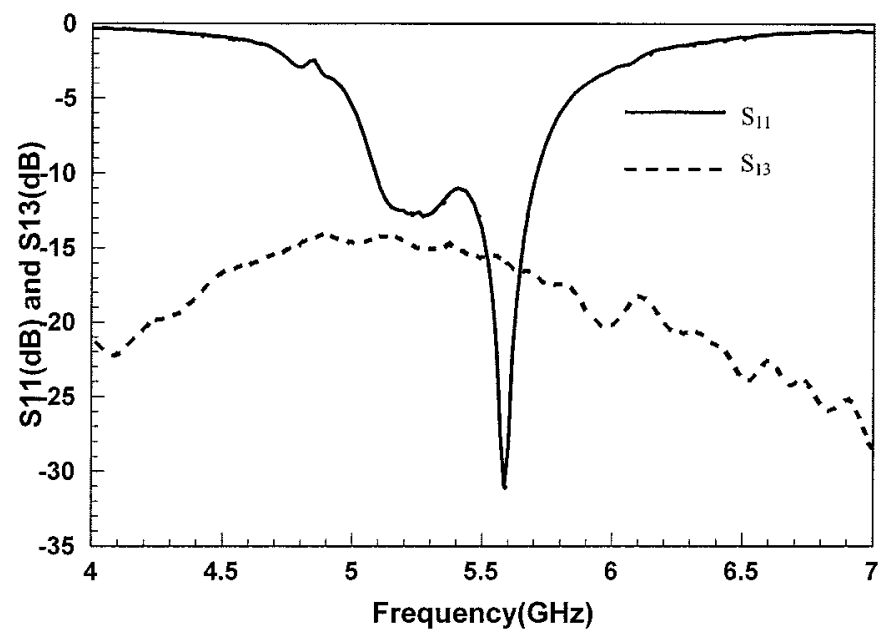

(b)

Fig. 13. (a) Simulated return loss and mutual coupling for the four-qdime antenna system. (b) Measured return loss and mutual coupling between two qdime antennas of a four qdime prototype.

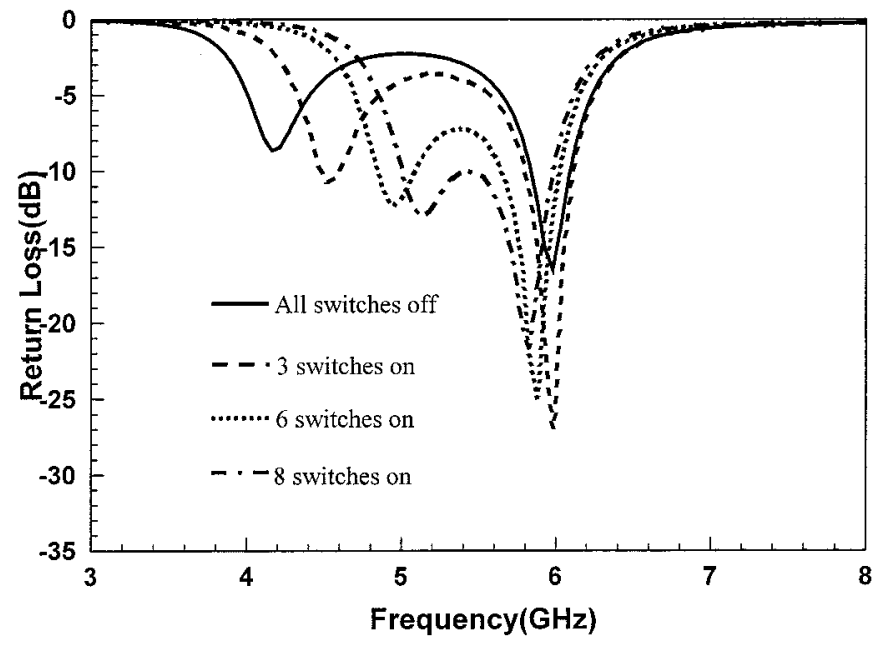

Fig. 14. Return loss for the MEMS switch-tunable qdime antenna.

position of a two-element antenna geometry. This analysis for the two-element geometry shows the capability of the antenna to function with separation distances as close as an inter-element center-to-center distance, in both E-plane and H-plane directions, smaller than $\lambda / 2$, with a spacing between antenna edges smaller than $0.1 \lambda$. Fig. 11 shows mutual coupling $\left(S_{12}\right)$ for different orientation angles $\alpha$. The interaction is lower when the antennas are oriented with $\alpha=90^{\circ}$, where an optimal electrical separation and field orthogonality is reached. For most communications applications, such as spatial or angular diversity techniques, low correlation coefficients between different radiating elements require mutual coupling below $15 \mathrm{~dB}$ [30], [31]. Our results show that for the actual interelement spacing any $\alpha$-orientation is suitable, allowing a variety of spatial geometries. Once the single- and two-element geometry were fully characterized, a planar set of four elements was designed for minimum space requirement, compatible with a mutual coupling figure below $15 \mathrm{~dB}$. Fig. 12 shows the actual dimensions of 


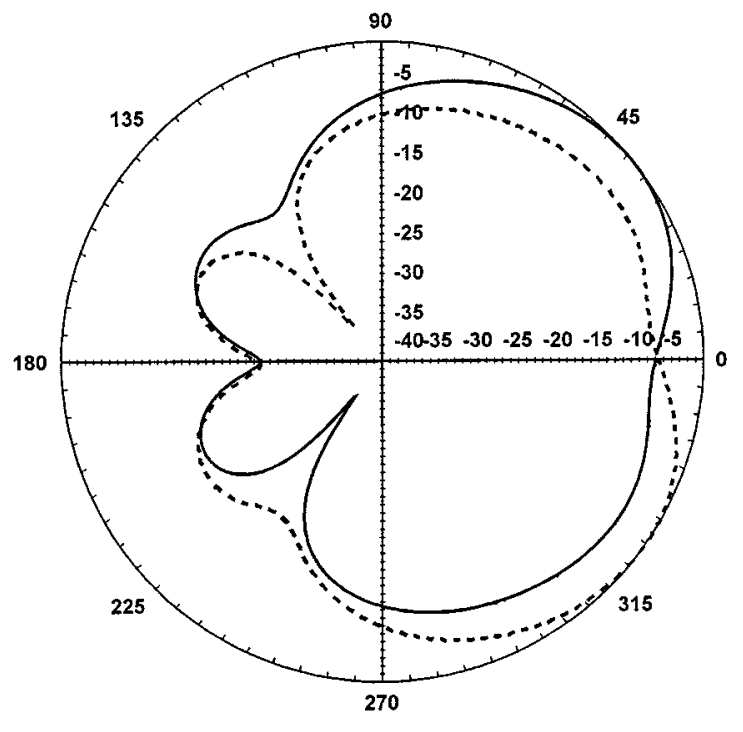

(a)

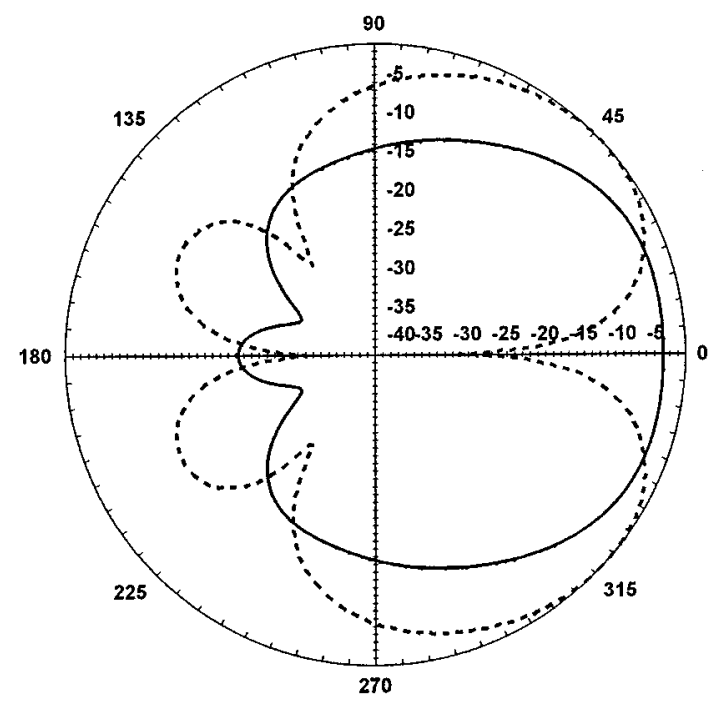

(b)

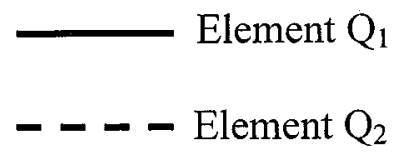

- - - Element $\mathrm{Q}_{2}$

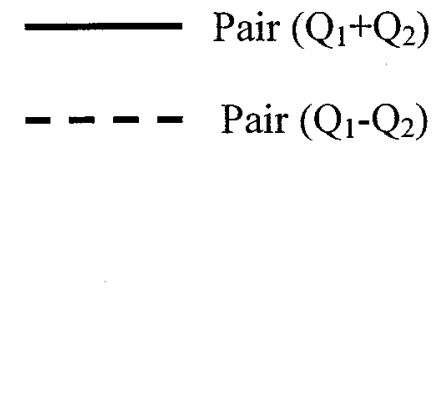

Fig. 15. Reconfigurable radiation pattern for the MEMS switch-tunable qdime antenna. (a) Copolar $x-z$ plane patterns corresponding to the $q$ dime elements $Q_{1}$ and $Q_{2}$. (b) Copolar $x-y$ plane patterns corresponding to the pair qdime element-combination $\left(Q_{1}+Q_{2}\right)$ and $\left(Q_{1}-Q_{2}\right)$.

the antenna for 5-6 GHz broad-band operation, with an overall dimension of $45 \times 45 \times 3.2 \mathrm{~mm}^{3}(0.8 \lambda \times 0.8 \lambda \times 0.06 \lambda$ at $5.5 \mathrm{GHz}$ ). Fig. 13(a) shows the simulated return loss and mutual coupling results for this geometry. In order to validate the simulation, a test prototype of the four qdime geometry of Fig. 12 was fabricated with a 0.3 -mm-thick copper sheet using the same etching and soldering technique as explained in Section II-C. Ports number 1 and 3 were fed by coaxial lines and ports number 2 and 4 properly terminated. Fig. 13(b) contains measured return loss of the port 1 and mutual coupling between ports 1 and 3 that show good agreement with the previous simulated results. A small discrepancy may have been caused by imperfect etching process.

To add frequency or pattern reconfigurability to the multi-element antenna, a set of MEMS switches can be strategically placed in different locations of the antenna structure. To study reconfigurability of antenna frequency range, either for dual or broad-band operation, we placed shorting strip lines $(60 \mu \mathrm{m} \times$ $200 \mu \mathrm{m}$ in size) representing MEMS switches designed to control the effective length of key-tuning elements. These lines were located at lower patch level, spanning from lower patch to the vertical wall so that we can tune the length of the lower slot by changing $w_{l}$. When the MEMS switch is activated, its electrically actuated mechanical membrane provides physical contact between lower patch and vertical wall. Fig. 14 shows the corresponding simulated results obtained by successively activating a specific number of switches. We show that this switching mechanism allows the operational frequency to be effectively tuned over a $40 \%$ bandwidth. On the other hand, to study the reconfigurability capabilities in terms of radiation pattern changes, a second set of MEMS switches were placed on the four-feeding lines (see Fig. 12). Using the four-antenna elements $Q_{i}$ by activating the four switches, a spatial $\left(Q_{1}, Q_{2}, Q_{3}\right.$, or $\left.Q_{4}\right)$, polarization $\left(Q_{1}+Q_{3}\right.$ or $\left.Q_{2}+Q_{4}\right)$ or a combined spatial and polariza- 
tion diversity system can then be obtained. Fig. 15(a) shows the copolar $x-z$ plane patterns radiated by the antenna element $Q_{1}$ and $Q_{2}$. An additional complementary behavior can be obtained when the four switches are combined with a $\lambda / 2$ phase shifter. Fig. 15(b) shows two different copolar $x-y$ plane simulated patterns that were created by sequentially feeding the antenna pair $\left(Q_{1}, Q_{2}\right)$ in-phase $\left(Q_{1}+Q_{2}\right)$ or out of phase $\left(Q_{1}-Q_{2}\right)$. The results for different radiation patterns show different degrees of pattern orthogonality, which make it suitable for radiation pattern diversity.

\section{CONCLUSION}

A novel miniature broad-band antenna structure having attractive impedance and radiation characteristics has been proposed for those communication applications where space volume of the antenna is very limited. An extensive and detailed numerical study has been performed to identify the effects of key antenna elements on its performance. The results of this parametric study were used to develop a general procedure to design different miniature dime antenna geometries for specific applications and for operation at a specific frequency range. A 5.2-GHz prototype based on calculated parameters was fabricated and measured. Very good agreement between measurement and simulation, for both input impedance and radiation pattern, verifies the accuracy of the simulation tool used as well as the robustness of the design procedure.

A slightly modified geometry was subsequently created and optimized for compact multi-element diversity antenna architecture. Finally antenna reconfigurability in terms of frequency range and radiation pattern capabilities was studied using a set of strategically located MEMS switches. A four-element prototype with an overall dimension of $0.8 \lambda \times 0.8 \lambda \times 0.06 \lambda$ and a frequency band operation in the Bluetooth range of $5-6 \mathrm{GHz}$ has been designed, simulated, and validated with a first experimental prototype.

\section{ACKNOWLEDGMENT}

The authors would like to thank N. G. Alexopoulos for helpful discussions on basic antenna parameters.

\section{REFERENCES}

[1] J. L. Pan, S. S. Rappaport, and P. M. Djuric, "A multibeam medium access scheme for multiple services in wireless cellular communications," in Proc. IEEE 1999 Int. Conf. Communication, vol. 3, 1999, pp. $1673-1677$.

[2] C. T. C. Nguyen, L. P. B. Katehi, and G. M. Rebeiz, "Micromachined devices for wireless communications," Proc. IEEE, vol. 86, pp. 1756-1768, Aug. 1998.

[3] E. R. Brown, "RF-MEMS switches for reconfigurable integrated circuits," IEEE Trans. Microwave Theory Tech., vol. 46, pp. 1868-1996, Nov. 1998.

[4] N. S. Barker and G. M. Rebeiz, "Distributed MEMS true-time delay phase shifters and wide-band switches," IEEE Trans. Microwave Theory Tech., vol. 46, pp. 1881-1890, Nov. 1998.

[5] F. De Flaviis, R. Coccioli, and T. Itoh, "Nonlinear analysis and evaluation of distorsion introduced by micro electromechanical switches in reconfigurable antenna systems," in Proc. AP2000, Millennium Conf. Antennas Propagat., Davos, Switzerland, Apr. 9-14, 2000.
[6] R. Garg, P. Barthia, I. Bahl, and A. Ittipibon, Microstrip Antenna Design book. Norwood, MA: Artech House, 2001.

[7] O. Staub, J. F. Zurcher, A. K. Skrivervik, and J. R. Mosig, "PCS antenna design: The challenge of miniaturization," in Proc. IEEE AP-S Int. Symp. 1999, vol. 1, pp. 548-551.

[8] H. K. Kan and R. B. Waterhouse, "Size reduction technique for shorted patches," Electron. Lett., vol. 35, no. 12, pp. 948-949, June 1999.

[9] M. Liebendorfer and U. Dersch, "Wireless LAN diversity antenna system for PCMIA card integration," in Proc. IEEE Vehicle Technology Conf., 1997, pp. 2022-2026.

[10] S. D. Targonski, R. B. Waterhouse, and D. M. Pozar, "Design of wide-band aperture-stacked patch microstrip antennas," IEEE Trans. Antennas Propagat., vol. 46, pp. 1245-1251, Sept. 1998.

[11] K. M. Luk, R. Chair, and K. F. Lee, "Small rectangular patch antenna," Electron. Lett., vol. 34, pp. 2366-2367, Dec. 1998.

[12] G. Kossiavas, A. Papiernick, J. P. Boisset, and M. Sauvan, "The C-patch: A small microstrip element," Electron. Lett., vol. 25, no. 4, pp. 253-254, Feb. 1989.

[13] X. X. Zhang and F. Yang, "Study of a slit cut on a microstrip antenna and its applications," Microwave Opt. Technol. Lett., vol. 18, no. 4, pp. 297-300, July 1998.

[14] J. H. Lu, C. L. Tang, and K. L. Wong, "Novel dual-frequency and broad-band designs of slot-loaded equilateral triangular microstrip antennas," IEEE Trans. Antennas Propagat., vol. 48, pp. 1048-1054, July 2000.

[15] P. Salonen, M. Keskilammi, and M. Kivikoski, "Single-feed dual-band planar inverted-F antenna with U-shaped slot," IEEE Trans. Antennas Propagat., vol. 48, pp. 1262-1264, Aug. 2000.

[16] R. R. Ramirez, H. Elsadek, L. Jofre, and F. De Flaviis, "Reduced size single and dual band linear polarized microstrip antennas for mobile communications," in Proc. IEEE AP-S Int. Symp., Boston, MA, July $8-13,2001$.

[17] R. B. Waterhouse, "Small microstrip patch antenna," Electron. Lett., vol. 31, pp. 604-605, 1995.

[18] R. B. Waterhouse, S. D. Targonski, and D. M. Kototoff, "Design and performance of small printed antennas," IEEE Trans. Antennas Propagat., vol. 46, pp. 1629-1633, Nov. 1998.

[19] T. K. Lo, "Miniature aperture-coupled microstrip antenna of very high permittivity," Electron. Lett., vol. 33, pp. 9-10, 1997.

[20] J. S. Colburn and Y. Rahmat-Samii, "Patch antennas on externally perforated high dielectric constant substrates," IEEE Trans. Antennas Propagat., vol. 47, pp. 1785-1794, Dec. 1999.

[21] J. S. McLean, "A re-examination of the fundamental limits on the radiation Q of electrically small antennas," IEEE Trans. Antennas Propagat., vol. 44, pp. 672-676, May 1996.

[22] D. R. Jackson and N. G. Alexopoulos, "Simple approximate formulas for input resistance, bandwidth and efficiency of a resonant rectangular path," IEEE Trans. Antennas Propagat., vol. 39, pp. 407-410, 1991.

[23] B. A. Cetiner, L. Jofre, and F. De Flaviis, "A packaged miniature antenna for wireless networking," in Proc. 34th Int. Symp. Microelectronics, Int. Microelectronics and Packaging Soc., IMAPS 2001, Baltimore, MD, Oct. 9-11, 2001.

[24] _ "A miniature broadband antenna for portable communications terminals," in IEEE AP-S Int. Symp., Boston, MA, July 8-13, 2001.

[25] K. Siwiak, Radiowave Propagat. Antennas for Personal Commun.. Norwood, MA: Artech House, 1998.

[26] O. Staub, J. F. Zurcher, and A. Skrivervik, "Some considerations on the correct measuremens of the gain and bandwidth of electrically small antennas," Microwave Opt. Technol. Lett., vol. 17, no. 3, pp. 156-160, Feb. 1998.

[27] R. Sorrentino and L. Roselli, "A new simple and accurate formula for microstrip radial stub," Trans. Microwave Guided Wave Lett., vol. 2, pp. 480-482, Dec. 1992.

[28] W. C. Jakes, Microwave Mobile Commun.. New York: IEEE Press, 1974.

[29] W. C. Y. Lee, Mobile Communications Engineering. New York: McGraw-Hill, 1998.

[30] J. S. Colburn, Y. R. Samii, M. A. Jensen, and G. J. Pottie, "Evaluation of personal communications dual-antenna handset diversity performance," IEEE Trans. Antennas Propagat., vol. 47, pp. 737-746, 1998.

[31] S. Tabbane, Handbook of Mobile Radio Networks. Norwood, MA: Artech House, 2000. 


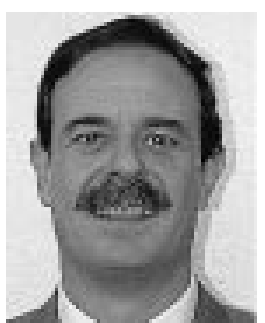

Luis Jofre (S'79-M'83) was born in Barcelona, Spain, in 1956. He received the M.Sc. (Ing) and $\mathrm{Ph} . \mathrm{D}$. (Doctor Ing.) degrees in electrical engineering (telecommunications eng.), from the Technical University in Catalonia (UPC), Barcelona, Spain, in 1978 and 1982, respectively.

From 1979 to 1980, he was Research Assistant in the Electrophysics Group at UPC, where he worked on the analysis and near-field measurement of antenna and scatterers. From 1981 to 1982 , he was with the Ecole Superieure d'Electricite, Paris, France, where he worked in microwave antenna design and imaging techniques for medical and industrial applications. In 1982, he was appointed Associate Professor at the Communications Department of the Telecommunication Engineering School at the Technical University of Catalonia (UPC), where he became Full Professor in 1989. From 1986 to 1987, he was a Visiting Fullbright Scholar at the Georgia Institute of Technology, Atlanta, working on antennas and electromagnetic imaging and visualization. From 1989 to 1994 he served as Director of the Telecommunication Engineering School (UPC), and from 1994 to 2000, as UPC Vice-Rector for Academic Planning. His research interests include antennas, scattering, electromagnetic imaging, and wireless communications, and he has published more than 100 scientific and technical papers, reports, and chapters in specialized volumes. During 2000 and 2001, he was on sabbatical leave from UPC, and was a Visiting Professor at the Electrical and Computer Engineering Department, Henry Samueli School of Engineering, University of California, Irvine, where he focused on antennas and systems miniaturization for wireless and sensing applications.

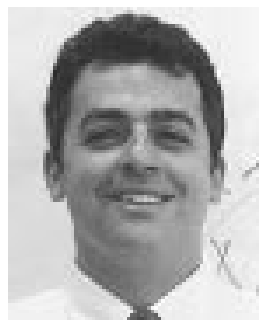

Bedri A. Cetiner (M'00) was born in Gazi Magosa (Famagusta), Cyprus in 1969. He received the Ph.D. degree in electronic and communication engineering from the Yildiz Technical University, Istanbul, Turkey, in 1999.

From November 1999 to June 2000, he was with the University of California, Los Angeles as a Visiting Scholar. Since June 2000, he has been with the Electrical and Computer Engineering Department, University of California, Irvine, where he is currently working as a postdoctoral researcher. His current research interests focus on the analysis and design of microwave circuits, application of MEMS technology for development of microwave devices and small antennas for wireless communication.

Dr. Cetiner is the recipient of the 1999 NATO scholarship given by The Scientific and Technical Research Council of Turkey (TUBITAK) for postdoctoral research.

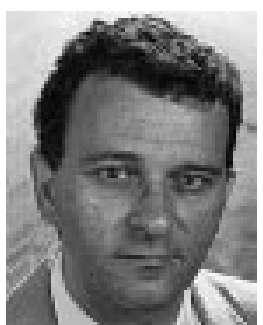

Franco De Flaviis was born in Teramo, Italy, in 1963. He received the degree in electronics engineering from the University of Ancona, Italy, and the M.S. and Ph.D. degrees in electrical engineering from the Department of Electrical Engineering at the University of California at Los Angeles (UCLA), in 1990, 1994, and 1997, respectively.

In 1991, he worked at Alcatel, Torino, Italy, as a Researcher specializing in the area of microwave mixer design. In 1992, he was a Visiting Researcher at UCLA, working on low-intermodulation mixers. In 1998, he joined the Department of Electrical and Computer Engineering, University of California, Irvine, as an Assistant Professor. He is presently working in the field of novel antenna designs for wireless systems. His research includes both theoretical and experimental studies of microwave mixers and circuits and the synthesis of low-Ioss ferroelectric material for phase-shifter design to be employed in scan-beam antenna systems. 